\title{
Identification of 8 INTEGRAL hard X-ray sources with Chandra
}

\author{
S. Sazonov ${ }^{1,2}$, E. Churazov ${ }^{1,2}$, M. Revnivtsev ${ }^{1,2}$, A. Vikhlinin ${ }^{3,2}$, and R. Sunyaev ${ }^{1,2}$ \\ ${ }^{1}$ Max-Planck-Institut für Astrophysik, Karl-Schwarzschild-Str. 1, 85740 Garching bei München, Germany \\ e-mail: sazonov@mpa-garching.mpg.de \\ 2 Space Research Institute, Russian Academy of Sciences, Profsoyuznaya 84/32, 117997 Moscow, Russia \\ 3 Harvard-Smithsonian Center for Astrophysics, 60 Garden St., Cambridge, MA 02138, USA
}

Received 29 August 2005 / Accepted 22 October 2005

\section{ABSTRACT}

We report the results of identification of 8 hard X-ray sources discovered by the INTEGRAL observatory during the ongoing all-sky survey. These sources have been observed by Chandra. In 6 cases a bright X-ray source was found within the INTEGRAL localization region, which permitted to unambiguously identify 5 of the objects with nearby galaxies, implying that they have an active galactic nucleus (AGN), whereas one source is likely an X-ray binary in LMC. 4 of the 5 newly discovered AGNs have measured redshifts in the range $0.025-0.055$. The $\mathrm{X}$-ray spectra reveal the presence of significant amounts of absorbing gas $\left(N_{\mathrm{H}}\right.$ in the range $\left.10^{22}-10^{24} \mathrm{~cm}^{-2}\right)$ in all 5 AGNs, demonstrating that INTEGRAL is starting to fill in the sample of nearby obscured AGNs.

Key words. surveys - galaxies: Seyfert - X-rays: general

\section{Introduction}

X-ray surveys have been playing a leading role in studying the demography and cosmological evolution of active galactic nuclei (AGNs). In particular, deep surveys performed in the standard X-ray band (2-10 keV) have dramatically improved our knowledge of AGNs at $z \gtrsim 0.3$ (e.g. Brandt \& Hasinger 2005). However, due to the small solid angle, deep surveys are not well suited for studying the local AGN population. Furthermore, there is sufficient evidence that most AGNs are heavily intrinsically obscured at optical and soft X-ray wavelengths. To obtain a full picture of the local AGN population there is therefore a need in very large area surveys performed at energies above $10 \mathrm{keV}$. Such surveys should also be useful for population studies of Galactic hard X-ray sources.

Recently, a high Galactic latitude $\left(|b|>10^{\circ}\right)$ survey of the whole sky in the 3-20 keV band was performed with the RXTE observatory (Revnivtsev et al. 2004; Sazonov \& Revnivtsev 2004), detecting in total $\sim 300$ sources, including $\sim 100$ AGNs. Even this survey was however strongly biased against sources with intrinsic absorption in excess of a few $10^{23} \mathrm{H}$ atoms per $\mathrm{cm}^{2}$.

A comparable or better (for strongly absorbed sources and/or in crowded regions) capability for studying bright ( $\gtrsim 1 \mathrm{mCrab}$ ) hard X-ray sources is now provided (e.g. Krivonos et al. 2005) by the IBIS telescope of the INTEGRAL observatory, owing to its sensitivity peaking above $20 \mathrm{keV}$, large field of view and good $\left(\sim 10^{\prime}\right)$ angular resolution. INTEGRAL has been observing multiple targets since October 2002 and more than half of the sky has been covered as of spring 2005. A recently started campaign to observe with INTEGRAL the remaining sky regions is to be completed in 2006. Once the survey of the whole sky is completed, it will be used for a statistical study of extragalactic hard X-ray sources. This will include the construction of the source number-flux function as well as the hard X-ray luminosity function and absorption column density distribution of local AGNs.

Although most of the INTEGRAL sources detected so far away from the Galactic plane and Galactic Center can be reliably associated with known AGNs, there is a significant fraction of unidentifed sources. We hope to identify most of these sources by improving their localization to few arcseconds using focusing X-ray telescope observations, so that optical identification then becomes straightforward. In the present pilot study we use Chandra to identify 8 hard X-ray sources discovered by INTEGRAL.

\section{Observations}

Public and our proprietary INTEGRAL/IBIS observations cover approximately $50 \%$ of the sky as of April 2005. We constructed a map of the observed sky in the 17-60 keV band from the data of the IBIS/ISGRI detector. The details of the analysis and the catalog of detected sources will be presented elsewhere, after new observations from the ongoing all-sky survey are added and analyzed.

For the present study we compiled a representative sample of 8 new hard X-ray sources, detected with statistical 

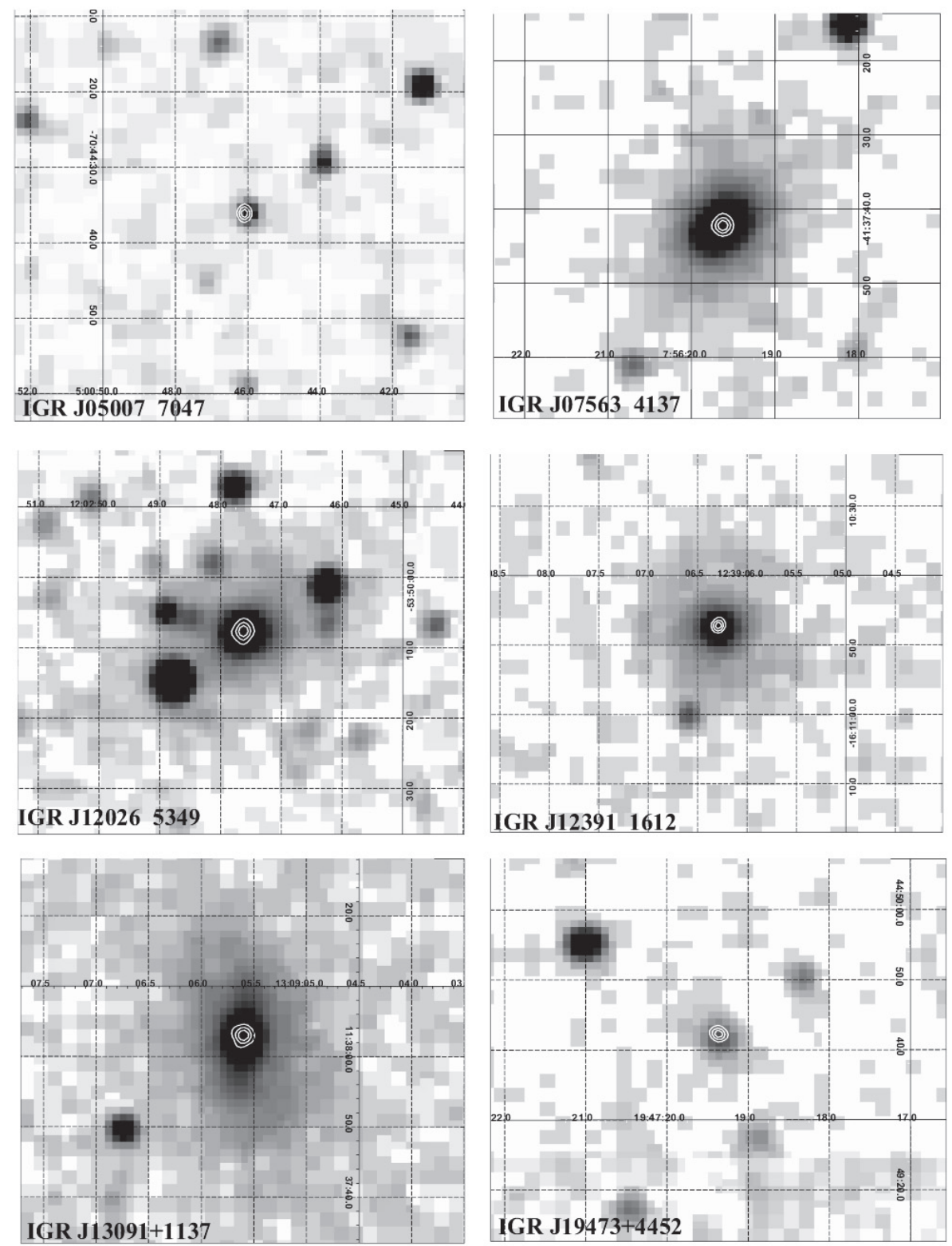

Fig. 1. Chandra X-ray brightness contours overlayed on the 2 MASS $J$-band images of 6 INTEGRAL sources. Each image $\left(\sim 0.8^{\prime} \times 0.8^{\prime}\right)$ falls into the original INTEGRAL localization region $\left(\sim 3^{\prime}\right.$ in radius, not shown).

significance higher than $5.5 \sigma$ on the summed INTEGRAL map. These sources are located significantly far away from both the Galactic plane $\left(>5^{\circ}\right)$ and Galactic Center $\left(>60^{\circ}\right)$, and were not detected in the Rosat All-Sky Survey (http://www. xray.mpe.mpg.de/cgi-bin/rosat/rosat-survey). The ISGRI localizations are $2-3^{\prime}$ radius ( $90 \%$ confidence). This INTEGRAL sample was observed by Chandra/ACIS-I as part of the DDT program in June-July 2005, with an exposure of $3.5 \mathrm{ks}$ per source.

\subsection{Identification}

In the Chandra fields for 6 INTEGRAL sources there is a bright point source $\left(\sim 0.1-0.2 \mathrm{cts} \mathrm{s}^{-1}\right)$ inside the ISGRI error region that can be unambiguously associated with the IGR source, since sources with corresponding X-ray fluxes (a few $\left.10^{-12} \mathrm{erg} \mathrm{cm}^{-2} \mathrm{~s}^{-1}\right)$ are quite rare $(\$ 1$ per $10 \mathrm{sq}$. deg.) at high Galactic latitude (e.g. Revnivtsev et al. 2004). In Fig. 1 the Chandra detections are shown on the near-infrared images from the Two Micron All-Sky Survey. Search around the accurate $\left(\sim 0.6^{\prime \prime}\right)$ Chandra positions in NED and SIMBAD reveals that 5 of the bright $X$-ray counterparts are located in the central regions of nearby galaxies, which together with the inferred high X-ray luminosities (see Table 2) implies that they are AGNs. For 3 of these galaxies there is a published redshift. We have measured the redshift of the 4th AGN, 2MASX J19471938+4449425, with the RTT-150 telescope (Bikmaev et al. 2005). The distance to 2MASX J07561963-4137420 remains unknown.

The position of the bright Chandra counterpart to IGR J05007-7047 coincides with the relatively bright, blue $(V=14.8, B-V=-0.01$, Massey 2002) star 
Table 1. Identification of INTEGRAL sources.

\begin{tabular}{|c|c|c|c|c|c|}
\hline \multicolumn{3}{|c|}{ INTEGRAL } & \multirow{2}{*}{$\begin{array}{c}\text { Chandra } \\
\alpha, \delta(2000)\end{array}$} & \multirow[t]{2}{*}{ Identification } & \multirow[t]{2}{*}{ Class of object } \\
\hline Name & $\alpha, \delta(2000)$ & Err & & & \\
\hline IGR J05007-7047 & $75.217-70.771$ & $\overline{3^{\prime}}$ & 05:00:46.08 - 70:44:36.0 & USNO-B1.0 0192-0057570 & HMXB in LMC? \\
\hline IGR J07563-4137 & $119.070-41.635$ & $3^{\prime}$ & $07: 56: 19.62-41: 37: 42.1$ & 2MASX J07561963-4137420 & AGN \\
\hline IGR J10252-6829 & $156.267-68.483$ & $3^{\prime}$ & $10: 25: 00.49-68: 27: 27.3$ & & \\
\hline IGR J11085-5100 & $167.144-51.014$ & $3^{\prime}$ & 11:08:50.48 -51:02:32.8 & USNO-B1.0 0389-0243808? & \\
\hline IGR J12026-5349 & $180.657-53.816$ & $3^{\prime}$ & 12:02:47.63 -53:50:07.7 & WKK 0560 & $\operatorname{AGN}(z$ \\
\hline IGR J12391-1612 & $189.780-16.196$ & $2^{\prime}$ & 12:39:06.29-16:10:47.1 & 2MASX J12390630-1610472 & $\operatorname{AGN}(z=0.0367)$ \\
\hline IGR J13091+1137 & $197.267+11.622$ & $3^{\prime}$ & 13:09:05.60 + 11:38:02.9 & NGC 4992 & $\operatorname{AGN}(z=0.0251)$ \\
\hline IGR J19473+4452 & $296.836+44.864$ & $3^{\prime}$ & $19: 47: 19.37+44: 49: 42.4$ & 2MASX J19471938+4449425 & $\operatorname{AGN}(z=0.0539)$ \\
\hline
\end{tabular}

Table 2. X-ray fluxes, luminosities and spectra.

\begin{tabular}{cccccccc}
\hline \hline Source & $\begin{array}{c}F(0.5-8 \mathrm{keV}) \\
10^{-12} \mathrm{erg} / \mathrm{cm}^{2} / \mathrm{s}\end{array}$ & $\begin{array}{c}L(0.5-8 \mathrm{keV})^{a} \\
\mathrm{erg} / \mathrm{s}\end{array}$ & $\begin{array}{c}\Gamma \\
\text { fixed }\end{array}$ & $\begin{array}{c}N_{\mathrm{H}}{ }^{b} \\
10^{22} \mathrm{~cm}^{-2}\end{array}$ & $\begin{array}{c}N_{\mathrm{H}, \mathrm{Gal}}{ }^{c} \\
F(17-60 \mathrm{keV}) \\
10^{-12} \mathrm{erg} / \mathrm{cm}^{2} / \mathrm{s}\end{array}$ & $\begin{array}{c}L(17-60 \mathrm{keV})^{a} \\
\mathrm{erg} / \mathrm{s}\end{array}$ \\
\hline IGR J05007-7047 & $3.0 \pm 0.3$ & $(9.1 \pm 0.9) \times 10^{35}$ & 1.4 & $1.0 \pm 0.2$ & 0.08 & $12 \pm 2$ & $(3.6 \pm 0.6) \times 10^{36}$ \\
IGR J07563-4137 & $3.5 \pm 0.4$ & - & 1.8 & $1.1 \pm 0.2$ & 0.43 & $15 \pm 2$ & - \\
IGR J12026-5349 & $6.7 \pm 0.9$ & $(1.1 \pm 0.1) \times 10^{43}$ & 1.8 & $2.2 \pm 0.3$ & 0.17 & $33 \pm 3$ & $(5.3 \pm 0.5) \times 10^{43}$ \\
IGR J12391-1612 & $2.0 \pm 0.3$ & $(5.5 \pm 0.8) \times 10^{42}$ & 1.8 & $1.9 \pm 0.3$ & 0.04 & $42 \pm 7$ & $(1.1 \pm 0.2) \times 10^{44}$ \\
IGR J13091+1137 & $1.2 \pm 0.2$ & $(1.5 \pm 0.3) \times 10^{42}$ & 1.8 & $90 \pm 10$ & 0.02 & $34 \pm 5$ & $(4.3 \pm 0.6) \times 10^{43}$ \\
IGR J19473+4452 & $3.0 \pm 1.0$ & $(1.8 \pm 0.6) \times 10^{43}$ & 1.8 & $11 \pm 1$ & 0.17 & $25 \pm 4$ & $(1.5 \pm 0.2) \times 10^{44}$ \\
\hline
\end{tabular}

${ }^{a}$ Observed luminosities, assuming $H_{0}=73 \mathrm{~km} \mathrm{~s}^{-1} \mathrm{Mpc}^{-1}$ for AGNs and $D=50 \mathrm{kpc}$ for IGR J05007-7047.

${ }^{b}$ Measured absorption column.

${ }^{c}$ Galactic absorption column based on Dickey \& Lockman (1990).

USNO-B1.0 0192-0057570. Based on its magnitude, color and positional coincidence with the Large Magellanic Cloud, we conclude that this object is very likely a high-mass $\mathrm{X}$-ray binary in LMC. With the luminosity $\sim 5 \times 10^{36} \mathrm{erg} \mathrm{s}^{-1}(1-60 \mathrm{keV}$, assuming a distance of $50 \mathrm{kpc}$ ), it is then the 4 th brightest HMXB in LMC (after LMC X-1, LMC X-3 and LMC X-4) according to INTEGRAL observations and historical data (e.g. Shtykovskiy \& Gilfanov 2005).

In either localization region of the 2 remaining sources, IGR J10252-6829 and IGR J11085-5100, there is a single relatively bright $\left(\sim 0.003\right.$ and $\sim 0.005 \mathrm{cts} \mathrm{s}^{-1}$, respectively) Chandra source, which might be associated with the IGR sources. However, the probability of the Chandra source occuring by chance inside the INTEGRAL error region is not small in both cases, as suggested by counts of extragalactic X-ray sources (e.g. Moretti et al. 2003) and by the fact that there are 5 (3) sources brighter than $0.0015 \mathrm{cts} \mathrm{s}^{-1}$ in the entire $\left(\sim 16^{\prime} \times 16^{\prime}\right)$ Chandra field for IGR J10252-6829 (IGR J11085-5100). Nonetheless, we provide the positions of the possible X-ray counterparts of both IGR sources in Table 1 and note that the possible counterpart of IGR J11085-5100 coincides with a weak $(B=20.3)$ star-like object. New INTEGRAL data suggest that both IGR J10252-6829 and IGR J11085-5100 may be transient sources since they were clearly detectable in 2004 but disappeared in 2005.

\subsection{X-ray spectra}

We next discuss the X-ray spectra of the IGR sources. Chandra provides information on the source spectra below $10 \mathrm{keV}$, while INTEGRAL yields the source fluxes in the $17-60 \mathrm{keV}$ band.
Two of the sources, IGR J12391-1612 and IGR J19473+4452, were also detected in the RXTE Slew Survey (Revnivtsev et al. 2004) where their fluxes in the $3-8 \mathrm{keV}$ and $8-20 \mathrm{keV}$ bands were measured. We show in Fig. 2 the resulting broad-band spectra for the 5 AGNs and the candidate HMXB in LMC.

The data for the 5 brightest Chandra sources suffer from photon pileup (e.g. Davis 2001). A generic signature of the pileup in the ACIS CCDs is photon migration from the good ( 0 , $2,3,4,6)$ to bad $(1,5,7)$ grade set (see Davis 2001, for details). The fraction of bad grade photons is negligible for non piledup sources. Therefore, the image regions strongly affected by pileup can be identified from the image containing only the bad grade photons. For our IGR sources, the affected regions are mostly confined to the central $1.5 \times 1.5 \mathrm{arcsec}$. Outside these regions, the pileup effects must be weak because the fraction of bad grade photons is small $(<10 \%)$. We based our spectral analysis for the piled-up sources on the source photons detected outside the central regions (i.e. excluding $\sim 80 \%$ of the PSF) and also restricted the analysis to energies below $5 \mathrm{keV}$. The nominal effective area calibration was corrected for the energy dependence of the flux scattered outside the region affected by pileup (http://cxc. harvard. edu/cal/Hrma/psf/). To construct the spectrum of IGR J13091+1137, unaffected by pileup, all photons from the whole PSF were used.

The Chandra spectra of all 5 AGNs are well approximated by a power law modified by photoabsorption along the line of sight. All 5 spectra exhibit a strong low-energy cutoff due to intrinsic absorption, with inferred column densities $\left(N_{\mathrm{H}}\right)$ ranging from $10^{22}$ to $10^{24} \mathrm{~cm}^{-2}$ (see Table 2). This explains the absence of soft X-ray counterparts in the Rosat All-Sky Survey. For all 5 spectra the photon index $(\Gamma)$ is constrained to the broad 


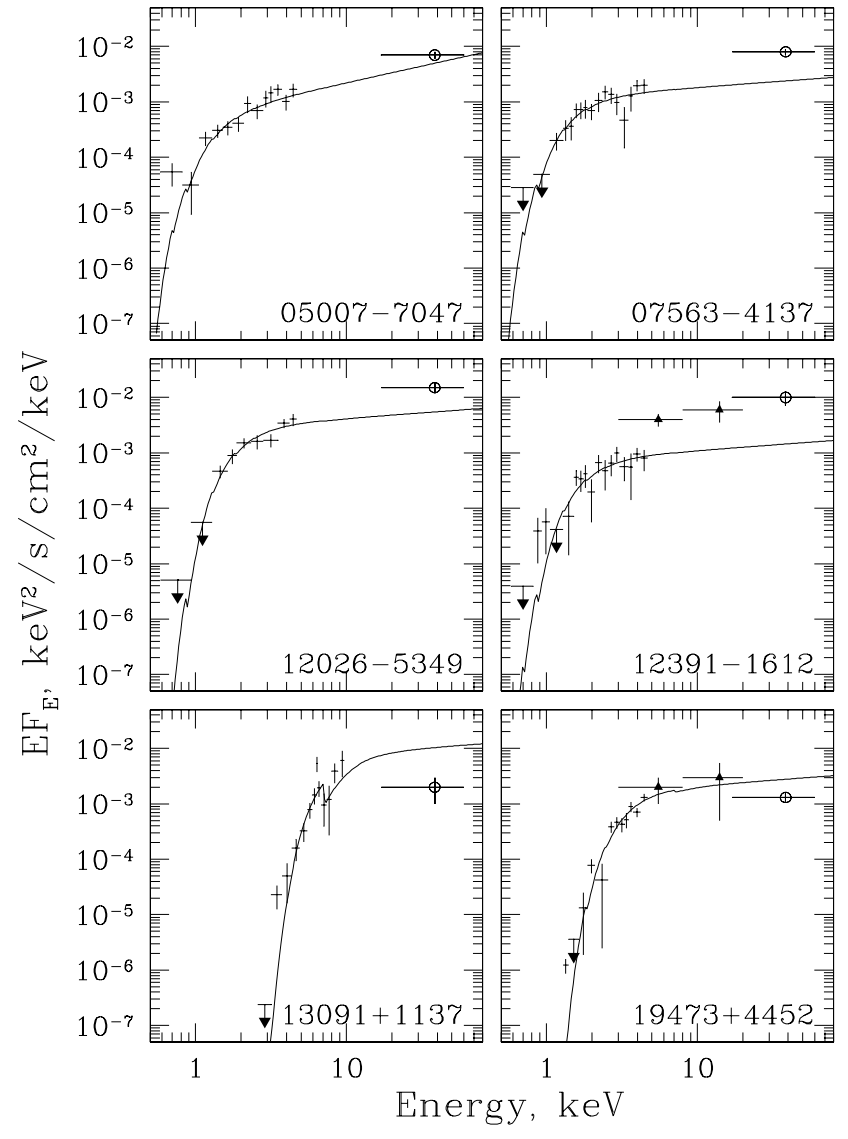

Fig. 2. Broad-band X-ray spectra of 6 IGR sources with bright Chandra counterparts. The data points between 0.5 and $5 \mathrm{keV}$ $(0.5-10 \mathrm{keV}$ for IGR J13091+1137) are from Chandra and the solid line is the best-fit model to these data (see Table 2) extrapolated to higher energies. Also shown are $17-60 \mathrm{keV}$ fluxes measured by INTEGRAL (open circles) and, for 2 sources, 3-8 keV and 8-20 keV fluxes measured by RXTE (filled triangles).

range 1.3-2.0. The best-fit models shown in Fig. 2 assume $\Gamma=1.8$, a value typical for AGNs. It can be seen that the extrapolated best-fit model misses the INTEGRAL and RXTE flux measurements by up to a factor of 5. This discrepancy likely results from a combination of uncertainty in the spectral slope and intrinsic source variability.

Similarly to the AGNs, the Chandra spectrum of IGR J05007-7047, the proposed LMC HMXB, is well fit by a power law ( $\Gamma$ in the range 1.3-2.0) modified by photoabsorption $\left(N_{\mathrm{H}} \approx 10^{22} \mathrm{~cm}^{-2}\right)$. In Fig. 2 we fixed $\Gamma$ at 1.4 , a value typical for HMXBs. The extrapolated best-fit model is in good agreement with the 17-60 keV flux measured by INTEGRAL.
Table 2 provides source fluxes at $0.5-8 \mathrm{keV}$ and $17-60 \mathrm{keV}$ as well as luminosities if the distance is known. The found AGN luminosities are typical for Seyfert galaxies.

\section{Discussion}

We observed with Chandra 8 hard X-ray sources discovered by INTEGRAL. 5 AGNs and a likely HMXB in LMC have been found. As expected, most of the new hard X-ray sources proved nearby $(z=0.025-0.055$ in the 4 cases with measured redshifts) AGNs with significant intrinsic absorption. This verifies our expectation of discovering a large number of obscured AGNs (mostly Seyfert 2 galaxies) during the INTEGRAL allsky survey. We note that INTEGRAL has also demonstrated its capability of discovering Seyfert 1 galaxies hidden behind the Milky Way (see e.g. Masetti et al. 2004).

This study demonstrates that the majority of hard X-ray sources discovered and localized by INTEGRAL at high Galactic latitude can be readily identified using short Chandra, XMM-Newton or SWIFT/XRT exposures.

Acknowledgements. This research is based on Chandra observations using Chandra Director's Discretionary Time. We are grateful to Harvey Tananbaum for his interest in the problem and useful discussions, and to William Forman for help. This research has made use of the SIMBAD database (operated at CDS, Strasbourg), NASA/IPAC Extragalactic Database and the Infrared Science Archive (operated by the Jet Propulsion Laboratory, California Institute of Technology), and data products of the 2MASS (joint project of the Univ. of Massachusetts and the IPAC, funded by the NASA and NSF).

\section{References}

Bikmaev, I., et al. 2005, in preparation

Brandt, W. N., \& Hasinger, G. 2005, ARA\&A, 43, 827

Davis, J. E. 2001, ApJ, 562, 575

Dickey, J. M., \& Lockman, F. J. 1990, ARA\&A, 28, 215

Krivonos, R., Vikhlinin, A., Churazov, E., et al. 2005, ApJ, 625, 89

Masetti, N., Palazzi, E., Bassani, L., Malizia, A., \& Stephen, J. B. 2004, A\&A, 426, L41

Massey, P. 2002, ApJS, 141, 81

Moretti, A., Campana, S., Lazzati, D., \& Tagliaferri, G. 2003, ApJ, 588, 696

Revnivtsev, M., Sazonov, S. Y., Jahoda, K., \& Gilfanov, M. 2004, A\&A, 418, 927

Sazonov, S. Y., \& Revnivtsev, M. G. 2004, A\&A, 423, 469

Shtykovskiy, P., \& Gilfanov, M. 2005, A\&A, 431, 597 